\title{
Application of Calorimetric Low Temperature Detectors (CLTD's) for Precise Stopping Power Measurements of Heavy Ions in Matter
}

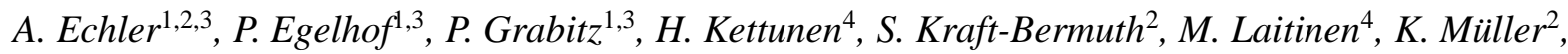 \\ M. Rossi ${ }^{4}$, W.H. Trzaska ${ }^{4}$, and A. Virtanen ${ }^{4}$ \\ ${ }^{1}$ GSI, Darmstadt, Germany; ${ }^{2}$ University of Gießen, Germany; ${ }^{3}$ University of Mainz, Germany; ${ }^{4}$ University of \\ Jyväskylä, Finland
}

Precise data on electronic stopping powers, i.e. specific energy loss $\mathrm{dE} / \mathrm{dx}$, for heavy ions are of high interest in various fields of research [1]. Unfortunately the accuracy of theoretical ab-initio $\mathrm{dE} / \mathrm{dx}$-calculations is nowadays still unsatisfactory. Therefore, predictions of stopping powers are usually calculated by semi-empirical computer codes, which are based on best fits to available experimental data. To improve the reliability of these calculations, precise $\mathrm{dE} / \mathrm{dx}$ data are needed for various projectile-target combinations and over a wide range of projectile energies, in particular for heavy projectiles at low energies, as measured data are scarce in that region [2]. However, for high ion masses and low energies ionization based energy detectors, that are commonly used in experiments for stopping power determination [3, 4], suffer from incomplete energy detection, resulting in pulse height defect and a relatively poor energy resolution. As CLTD's provide substantially better energy resolution and linearity for heavy ion detection, with the absence of any pulse height defect [5, 6], this type of energy detectors has the potential to increase sensitivity and accuracy for $\mathrm{dE} / \mathrm{dx}$ measurements and to extend the accessible energy range towards lower energies.

For that purpose a CLTD array, that has been developed at GSI in the past years $[5,6,7]$, has been used to replace the Si-detector in an established setup for $\mathrm{dE} / \mathrm{dx}$ measurements at the K-130 cyclotron at the University of Jyväskylä $[3,8]$, and to perform measurements with $0.05-1 \mathrm{MeV} / \mathrm{u}$ ${ }^{131} \mathrm{Xe}$-ions in different absorber materials. As an example the measured electronic stopping power of Xe ions in carbon is displayed in fig. 1. A summary of all results for carbon as well as nickel and gold absorbers can be found in [9], where also a more detailed description of the experiment is given. The obtained $\mathrm{dE} / \mathrm{dx}$ values show in general a good agreement with previously measured data in the higher energy range for all studied absorber materials, what provides a consistency check of the new experimental setup. Moreover the application of CLTD's for the present investigations allowed to considerably reduce calibration errors as compared to previous measurements with Si-detectors [10], and to extend the datasets to lower energies, not accessible before.

\section{References}

[1] ICRU Report 73, Journal of the ICRU 5, No 1 (2005).

[2] H. Paul, web site: www.exphys.jku.at/stopping/

[3] W.H. Trzaska et al., Nucl. Instr. Meth. B 195, 147, (2002).

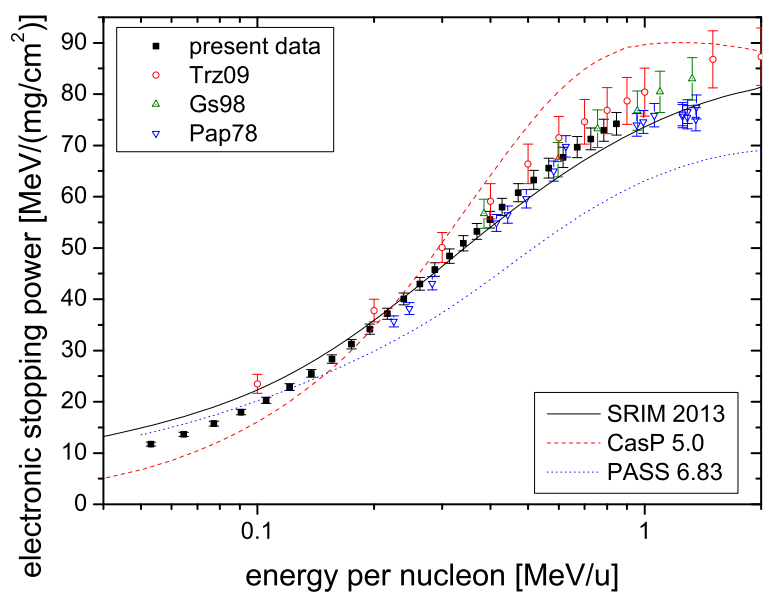

Figure 1: Electronic stopping power of Xe ions in carbon obtained from the present experiment [9] together with literature values that have been taken from the electronic library for stopping powers of H. Paul [2] (Trz09 [10], Gs98 [11], Pap78 [12]). The lines represent theoretical calculations with the semi-empirical computer code SRIM [13] and the ab-initio models CasP [14] and PASS [15].

[4] J. Zhang, Nucl. Instr. Meth. B 196, 1, (2002).

[5] P. Egelhof and S. Kraft-Bermuth, Topics in Applied Physics 99, 469, (2005).

[6] S. Kraft-Bermuth et al., Rev. Sci. Instrum. 80, 103304, (2009).

[7] A. Echler et al., J. Low Temp. Phys. 167, 5-6, 949, (2012).

[8] A. Javanainen et al., IEEE Trans. Nuc. Sci. 54, 4, 1158, (2007).

[9] A. Echler et al., J. Low Temp. Phys. DOI 10.1007/s10909013-1043-y (2014).

[10] W.H. Trzaska et al., Nucl. Instr. Meth. B 267, 3403, (2009).

[11] H. Geissel et al., various data summarized in electronic library of H. Paul [2].

[12] H. Pape et al., Zeitschr. f. Phys. A 286, 159, (1978).

[13] J.F. Ziegler, Nucl. Instr. Meth. B 268, 11-12, 1818, (2010), www.srim.org.

[14] G. Schiwietz and P.L. Grande, Nucl. Instr. Meth. B 273, 1, (2012), CasP download: http://www.casp-program.org/

[15] PASS-code based on Binary theory of electronic stopping, P. Sigmund and A. Schinner, Nucl. Instr. Meth. B 195, 64, (2002) 